\title{
Transcriptomic comparison between developing seeds of yellow- and black- seeded Brassica napus reveals that genes influence seed quality
}

\author{
Jinjin Jiang ${ }^{1}$, Shuang Zhu' ${ }^{1}$ Yi Yuan'1, Yue Wang ${ }^{1}$, Lei Zeng ${ }^{1}$, Jacqueline Batley ${ }^{2}$ and You-Ping Wang ${ }^{\text {1* }}$
}

\begin{abstract}
Background: Brassica napus is of substantial economic value for vegetable oil, biofuel, and animal fodder production. The breeding of yellow-seeded B. napus to improve seed quality with higher oil content, improved oil and meal quality with fewer antinutrients merits attention. Screening the genes related to this phenotype is valuable for future rapeseed breeding.

Results: A total of 85,407 genes, including 4317 novel genes, were identified in the developing seeds of yellow- and black-seeded B. napus, and yellow rapeseed was shown to be an introgression line between black-seeded B. napus and yellow-seeded Sinapis alba. A total of 15,251 differentially expressed genes (DEGs) were identified among all the libraries, and 563 and 397 common DEGs were identified throughout black and yellow seed development, including 80 upregulated and 151 downregulated genes related to seed development and fatty acid accumulation. In addition, 11 up-DEGs and 31 down-DEGs were identified in all developmental stages of yellow rapeseed compared with black seed. Enrichment analysis revealed that many DEGs were involved in biosynthetic processes, pigment metabolism, and oxidation-reduction processes, such as flavonoid and phenylpropanoid biosynthesis, phenylalanine metabolism, flavone and flavonol biosynthesis, and fatty acid biosynthesis and metabolism. We found that more than 77 DEGs were related to flavonoid and lignin biosynthesis, including $4 C L, C 4 H$, and $P A L$, which participated in phenylalanine metabolism, and BAN, CHI/TTS, DFR, F3H, FLS, LDOX, PAP, CHS/TT4, TT5, bHLH/TT8, WD4O, MYB, TCP, and CYP, which were involved in flavonoid biosynthesis. Most of these DEGs were downregulated in yellow rapeseed and were consistent with the decreased flavonoid and lignin contents. Both up- and down-DEGs related to fatty acid biosynthesis and metabolism were also analyzed, which could help to explain the improved oil content of yellow rapeseed.
\end{abstract}

Conclusion: This research provided comprehensive transcriptome data for yellow-seeded B. napus with a unique genetic background, and all the DEGs in comparison with the black-seeded counterpart could help to explain seed quality differences, such as lower pigmentation and lignin contents, and higher oil content.

Keywords: Brassica napus, Seed coat color, Flavonoid biosynthesis, Fatty acid, Gene expression

\footnotetext{
*Correspondence: wangyp@yzu.edu.cn

${ }^{1}$ Jiangsu Provincial Key Laboratory of Crop Genetics and Physiology,

Yangzhou University, Yangzhou 225009, China

Full list of author information is available at the end of the article
}

(c) The Author(s). 2019 Open Access This article is distributed under the terms of the Creative Commons Attribution 4.0 International License (http://creativecommons.org/licenses/by/4.0/), which permits unrestricted use, distribution, and reproduction in any medium, provided you give appropriate credit to the original author(s) and the source, provide a link to the Creative Commons license, and indicate if changes were made. The Creative Commons Public Domain Dedication waiver (http://creativecommons.org/publicdomain/zero/1.0/) applies to the data made available in this article, unless otherwise stated. 


\section{Background}

Brassica napus, as the third leading oilseed crop grown worldwide, greatly contributes to providing edible oils, biofuels, and animal fodder [1]. Rapeseed with increased oil content, better oil and meal quality, and improved yield has been the main breeding drive during the past decades [2]. Yellow-seeded B. napus was improved with better seed oil and meal quality due to fewer pigments and polyphenols; thus, breeding yellow rapeseed has been preferred for decades, and studies on the molecular mechanism of this phenotype have been reported as well [3]. Fewer antinutrients, including phenolic compounds, tannins, proanthocyanins (PAs), and lignin, in yellow rapeseed were correlated with the flavonoid biosynthetic, phenylpropanoid, and phenylalanine/tyrosine metabolic pathways with common substrates, such as coumaroyl CoA, caffeoyl CoA, and feruloyl CoA [4-6]. Hitherto, yellow-seeded $B$. napus has been selected from interspecific hybridization of Brassica species and intergeneric hybridization [7, 8]. As reported in Arabidopsis thaliana, mutations in transparent testa (TT) genes are responsible for seed coat color variations, including early (EBGs) and late (LBGs) biosynthesis genes [9]. The EBGs include TT4/CHS, TT5/CHI, TT6/F3H, and TT7/F3'H, and LBGs include TT3/DFR, TT18/LDOX/ANS, BAN/ ANR, TT12, TT19/GSTF12/GST26, and AHA10. In addition, the MYB-bHLH-WD40 (MBW) complex has been reported to have important regulatory functions in anthocyanin accumulation. Four MBW have been reported, including TT2-TT8-TTG1, MYB5-TT8-TTG1, TT2-EGL3-TTG1, and TT2-GL3-TTG1 [10]. Appelhagen et al. (2011) found that PAP1/MYB75 and PAP2/ MYB90 participated in the formation of MBW [11]. Homologous TTs related to seed coat variation in Brassicaceae have been delineated, including 95 copies of 21 TTs in B. napus [3]. Previously, F3'H, TT2, PAL, BAN, TTG1, TT10, and TT1 were cloned and shown to have functions in flavonoid biosynthesis [12-19]. Yu (2013) reviewed the molecular mechanism of manupulating seed coat color in Brassica species, including the homologous TTs cloned in Brassicas [20]. Besides, molecular markers have also been developed for yellow-seeded $B$. rapa [21-23], B. juncea [24, 25] and B. napus. Liu et al. (2012) reported a lignin biosynthesis gene, $B n C C R 1$, associated with yellow seed character, indicating a strong correlation between seed color and lignin content [26]. Stein et al. (2013) found that BnAHA10 had effects on seed color and lignin content [27]. Wang et al. (2017) identified 22 single nucleotide polymorphisms (SNPs) on 7 chromosomes associated with seed coat color using a genome wide association study (GWAS) [28]. Functional analysis of BnTT10 and BnTT1 revealed that they are involved in PA metabolism, lignin synthesis, seed coat pigmentation, and fatty acid (FA) biosynthesis [18, 19].
However, due to the genome complexity of B. napus and the sensitivity of yellow seed color to environmental influences (e.g., light, temperature, fertilizers, and harvesting time), the molecular mechanisms of this phenotype remained unclear until now $[29,30]$.

The short domestication history of B. napus after interspecific hybridization between $B$. rapa and $B$. oleracea and the artificial selection during rapeseed breeding have greatly narrowed the genetic background [31]. No yellow-seed germplasm has been found in natural $B$. napus, and most of the yellow-seeded $B$. napus were created by crossing between Brassica species, such as hybrids between $B$. napus and $B$. juncea, $B$. napus and $B$. carinata, $B$. juncea and B. oleracea [32, 33]. Wild species in Brassicaceae, such as Sinapis alba, S. arvensis, Camelina sativa, Crambe abyssinica, and Descurainia sophia, exhibit many desirable characteristics, such as yellow seed coat, high erucic acid, pod shattering resistance, high unsaturated FA contents, and resistance to various diseases and abiotic stresses [34-36]. Introducing gene resources from these wild species would help to enrich the genetic background of $B$. napus, accompanying desirable traits. Zhang et al. (2009) obtained yellow-seeded rapeseed from intergeneric hybrids between $B$. napus and D. sophia [37]. Previously, Wang et al. (2005) created somatic hybrids between B. napus and S. alba [38] and selected yellow-seeded B. napus from hybrid progenies with improved oil content and decreased antinutrients $[8,39,40]$. In the present study, we used RNA-Seq to identify the expression differences at different seed developmental stages of yellow- and black-seeded $B$. napus, revealing the expression patterns of genes involved in various biological pathways related to seed coat pigmentation, FA biosynthesis and metabolism.

\section{Results}

Transcriptome sequencing and read mapping of yellowand black-seeded $B$. napus

RNA-Seq was performed at three weeks after flowering (3 WAF), 4 WAF, 5 WAF, 6 WAF, and mature stage of yellow- and black-seeded B. napus to investigate the transcriptome difference that might be related to the quality variation between two rapeseeds. After quality control, 30.3 to $\sim 37.6$ million reads from the libraries were uniquely mapped to the B. napus genome (Table 1 ). A total of 85,407 genes, including 4317 novel genes, were expressed in the developing seeds of two rapeseeds (Fig. 1, Additional file 1: Table S1). We found 52,452 and 51,777 common genes were expressed in the developing seeds of yellow and black rapeseed, respectively. In addition, 67,629 (3 WAF), 64,070 (4 WAF), 64,775 (5 WAF), 62,143 (6 WAF), and 54,257 (mature stage) overlapping genes were expressed at each stage in two rapeseed lines. 
Table 1 Summary of read mapping for RNA-seq

\begin{tabular}{lllllllllll}
\hline $\begin{array}{l}\text { Sample } \\
\text { name }\end{array}$ & B3 & B4 & B5 & B6 & BM & Y3 & Y4 & Y5 & Y6 \\
\hline Raw reads & $47,536,866$ & $42,753,820$ & $44,341,556$ & $51,507,518$ & $51,272,946$ & $48,704,282$ & $45,694,038$ & $44,797,562$ & $45,996,584$ & $52,804,244$ \\
Clean & $45,555,018$ & $41,801,216$ & $40,620,518$ & $43,833,034$ & $48,665,348$ & $46,813,976$ & $43,798,322$ & $42,843,336$ & $43,736,106$ & $50,268,482$ \\
reads & & & & & & & & & \\
Total & $36,499,205$ & $34,214,293$ & $32,844,328$ & $35,032,820$ & $39,325,179$ & $38,116,991$ & $35,893,586$ & $35,376,347$ & $36,029,106$ & $40,839,634$ \\
mapped & $(80.12 \%)$ & $(81.85 \%)$ & $(80.86 \%)$ & $(79.92 \%)$ & $(80.81 \%)$ & $(81.42 \%)$ & $(81.95 \%)$ & $(82.57 \%)$ & $(82.38 \%)$ & $(81.24 \%)$ \\
Multiple & $2,175,877$ & $2,332,136$ & $2,561,972$ & $3,271,514$ & $3,151,365$ & $2,327,452$ & $2,287,911$ & $2,274,889$ & $2,451,932$ & $3,198,280$ \\
mapped & $(4.78 \%)$ & $(5.58 \%)$ & $(6.31 \%)$ & $(7.46 \%)$ & $(6.48 \%)$ & $(4.97 \%)$ & $(5.22 \%)$ & $(5.31 \%)$ & $(5.61 \%)$ & $(6.36 \%)$ \\
Uniquely & $34,323,328$ & $31,882,157$ & $30,282,356$ & $31,761,306$ & $36,173,814$ & $35,789,539$ & $33,605,675$ & $33,101,458$ & $33,577,174$ & $37,641,354$ \\
mapped & $(75.34 \%)$ & $(76.27 \%)$ & $(74.55 \%)$ & $(72.46 \%)$ & $(74.33 \%)$ & $(76.45 \%)$ & $(76.73 \%)$ & $(77.26 \%)$ & $(76.77 \%)$ & $(74.88 \%)$ \\
Reads map & $17,137,064$ & $15,873,247$ & $15,138,033$ & $15,900,632$ & $18,123,297$ & $17,872,927$ & $16,769,787$ & $16,508,902$ & $16,764,771$ & $18,866,098$ \\
to '+' & $(37.62 \%)$ & $(37.97 \%)$ & $(37.27 \%)$ & $(36.28 \%)$ & $(37.24 \%)$ & $(38.18 \%)$ & $(38.29 \%)$ & $(38.53 \%)$ & $(38.33 \%)$ & $(37.53 \%)$ \\
Reads map & $17,186,264$ & $16,008,910$ & $15,144,323$ & $15,860,674$ & $18,050,517$ & $17,916,612$ & $16,835,888$ & $16,592,556$ & $16,812,403$ & $18,775,256$ \\
to '-' & $(37.73 \%)$ & $(38.3 \%)$ & $(37.28 \%)$ & $(36.18 \%)$ & $(37.09 \%)$ & $(38.27 \%)$ & $(38.44 \%)$ & $(38.73 \%)$ & $(38.44 \%)$ & $(37.35 \%)$ \\
Non-splice & $21,266,684$ & $21,368,313$ & $21,381,177$ & $22,323,651$ & $24,534,251$ & $22,491,873$ & $22,291,894$ & $24,114,006$ & $24,559,779$ & $26,061,989$ \\
reads & $(46.68 \%)$ & $(51.12 \%)$ & $(52.64 \%)$ & $(50.93 \%)$ & $(50.41 \%)$ & $(48.05 \%)$ & $(50.9 \%)$ & $(56.28 \%)$ & $(56.15 \%)$ & $(51.85 \%)$ \\
Splice & $13,056,644$ & $10,513,844$ & $8,901,179$ & $9,437,655$ & $11,639,563$ & $13,297,666$ & $11,313,781$ & $8,987,452$ & $9,017,395$ & $11,579,365$ \\
reads & $(28.66 \%)$ & $(25.15 \%)$ & $(21.91 \%)$ & $(21.53 \%)$ & $(23.92 \%)$ & $(28.41 \%)$ & $(25.83 \%)$ & $(20.98 \%)$ & $(20.62 \%)$ & $(23.04 \%)$ \\
\hline
\end{tabular}

B3, B4, B5, B6 and BM indicate $3 \sim 6$ WAF and mature black seed. Y3, Y4, Y5, Y6 and YM indicate $3 \sim 6$ WAF and mature yellow seed

Besides, 47,912 genes were coexpressed in all the developmental stages of yellow and black rapeseed lines.

\section{Identification of differentially expressed genes}

Global changes in differentially expressed genes were identified using DESeq, and a total of 15,251 DEGs (including 523 novel genes) were identified among all the libraries. We found 563 common DEGs in B4 (4 WAF of black seed), B5 (5 WAF of black seed), B6 (6 WAF of black seed), and BM (mature stage of black seed) compared with B3 (3 WAF of black seed), including 141 upregulated and 331 downregulated genes at each developmental stage of the black seed. A total of 397 common DEGs were identified in Y4 (4 WAF of yellow seed), Y5 (5 WAF of yellow seed), Y6 (6 WAF of yellow seed), and YM (mature stage of yellow seed) compared with Y3 (3 WAF of yellow seed), including 92 upregulated and 220 downregulated genes throughout yellow seed development (Fig. 2a, b; Additional file 2: Table S2). Generally, 80 upregulated and 151 downregulated genes were identified during the seed development of two $B$. napus lines, which might be related to seed development and FA accumulation (Additional file 3: Table S3). Those DEGs related to cruciferin, oleosin, caleosin, late embryogenesis abundant (LEA) hydroxyproline, lipid transfer protein, myrosinase-binding protein, embryo-specific protein, and alpha-tonoplast intrinsic protein were upregulated, and those related to galactosyltransferase, glycosyl hydrolase, lipid transfer protein, basic leucine zipper protein, cytochrome P450 (CYP), laccase, chalcone synthase, delta vacuolar processing enzyme, jasmonic acid carboxyl

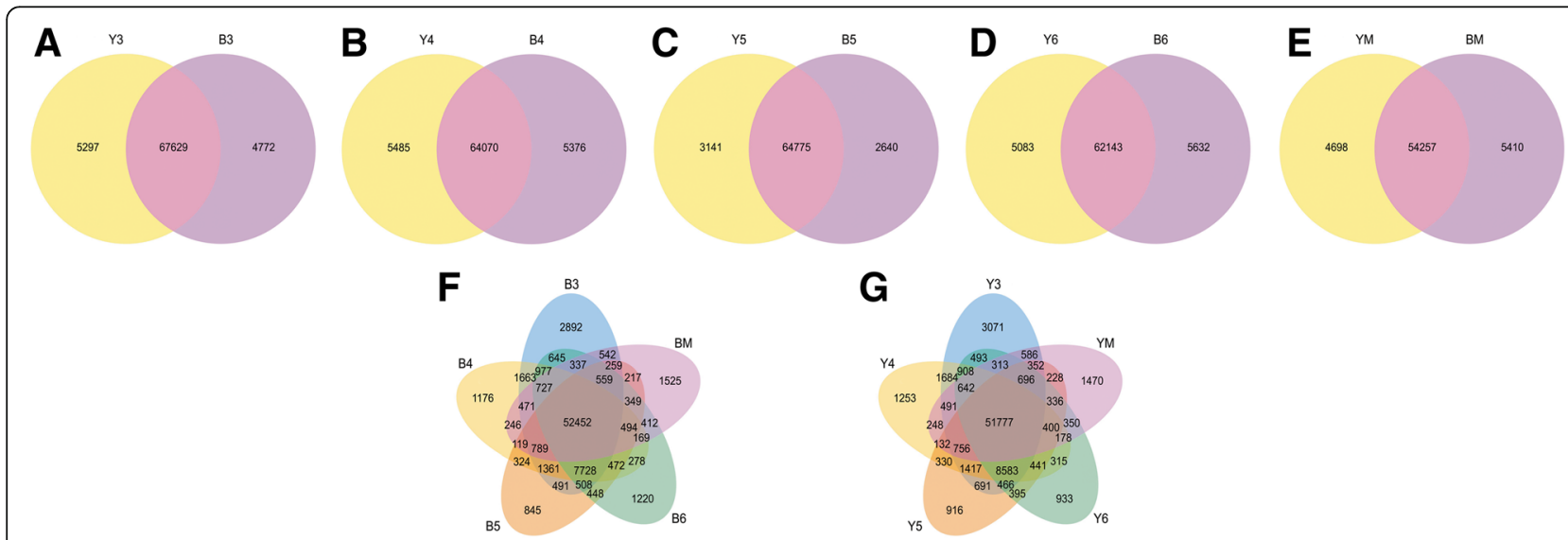

Fig. 1 Genes commonly and specifically expressed in developing seeds of yellow- and black-seeded B. napus. a-e Venn diagram of genes expressed between each developmental stage of yellow and black seed. $\mathbf{f}$ Venn diagram of genes in black seed. $\mathbf{g}$ Venn diagram of genes in yellow seed. B3, B4, B5, B6 and BM indicate $3 \sim 6$ WAF and mature black seed. Y3, Y4, Y5, Y6 and YM indicate $3 \sim 6$ WAF and mature yellow seed 


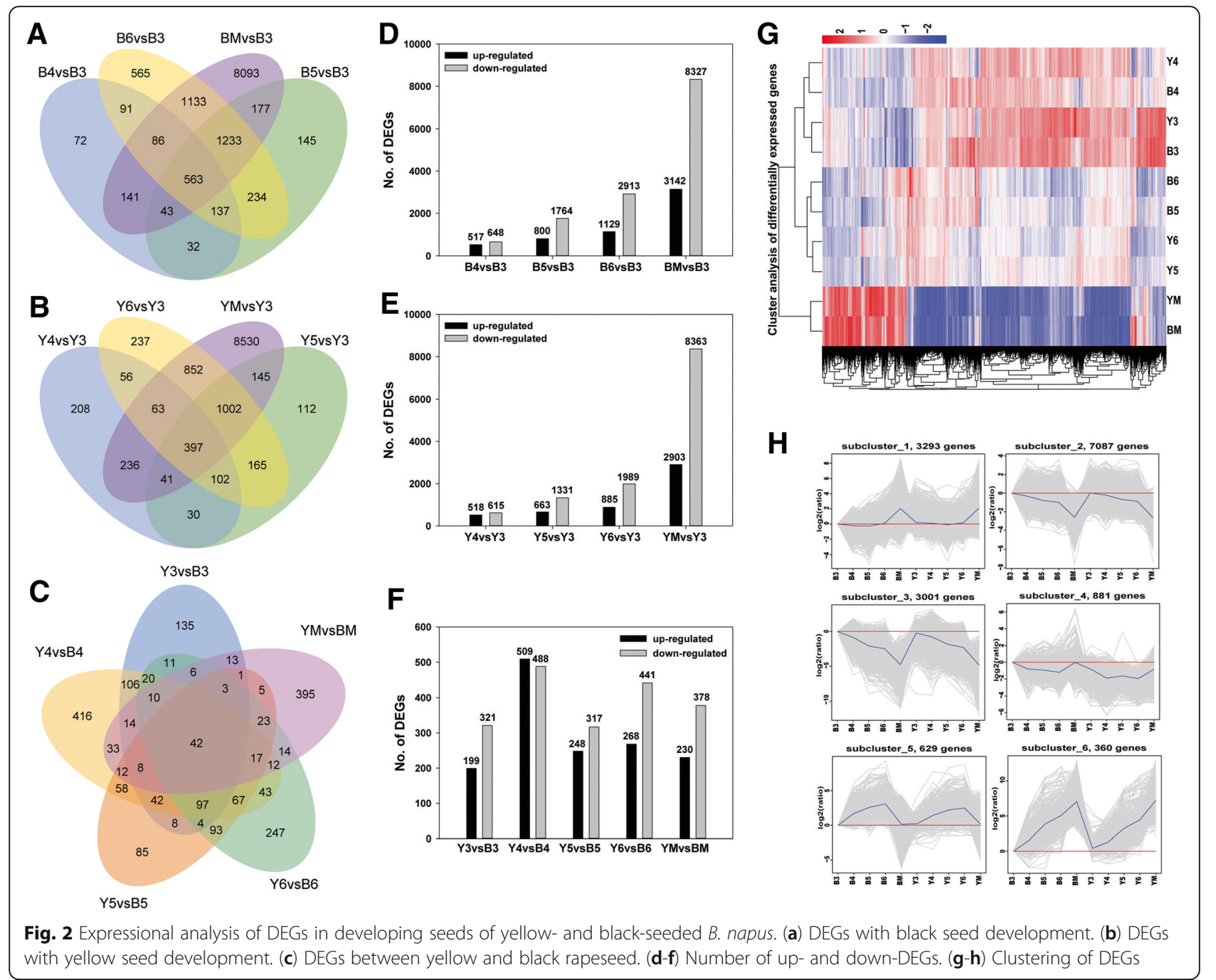

methyltransferase, and senescence were downregulated with seed development. In addition, 42 DEGs were identified between yellow and black rapeseeds at each stage, including 11 upregulated and 31 downregulated genes in yellow-seeded B. napus (Fig. 2c, Additional file 4: Table S4). These common DEGs were mainly related to the RNA-binding family protein, nascent polypeptide-associated complex (NAC), calcium binding EF-hand family protein, and metallothionein protein that were upregulated and dormancy/auxin associated protein, $\mathrm{S}$-adenosylmethionine synthetase, pectin lyase-like superfamily protein, insulinase family protein that were downregulated in all stages of yellow seed compared with black seed. The number of DEGs increased with seed development in both rapeseed lines, whereas the downregulated DEGs at the mature stage dramatically increased to 8000 (Fig. 2d, e). The up-regulated and downregulated DEGs between Y4 and B4 were higher than those in the other stages of the two rapeseed lines (Fig. 2f). Hierarchical cluster analysis of all the DEGs was performed using the $\log _{10}(\mathrm{RPKM}+1)$ value (Fig. 2g). H-cluster and SOM-cluster of the DEGs from five developmental stages of two rapeseed lines were also performed using the $\log _{2}$ (Fold change), and the DEGs in all the clusters showed similar patterns between two rapeseed lines, except for subcluster_1_1, which includes DEGs with significant differences between Y4 and B4 (Fig. 2h, Additional file 5: Figure S1).

\section{Enrichment analysis of DEGs}

To acknowledge the putative functions and pathways of the DEGs, GO and KEGG enrichment analysis of DEGs was proceeded with GOseq and KOBAS 2.0. Among all the identified DEGs, 11,772 were annotated with GO terms and assigned to three categories: cellular component, biological process, and molecular function. Most of the upand downregulated DEGs between the same stage of yellow and black seeds were assigned to catalytic activity, binding, metabolic process, cellular process, followed by cell, cell part, membrane, organelle part, macromolecular complex, regulation of biological process, and biological regulation (Fig. 3, Additional file 6: Table S5). In detail, among all the 


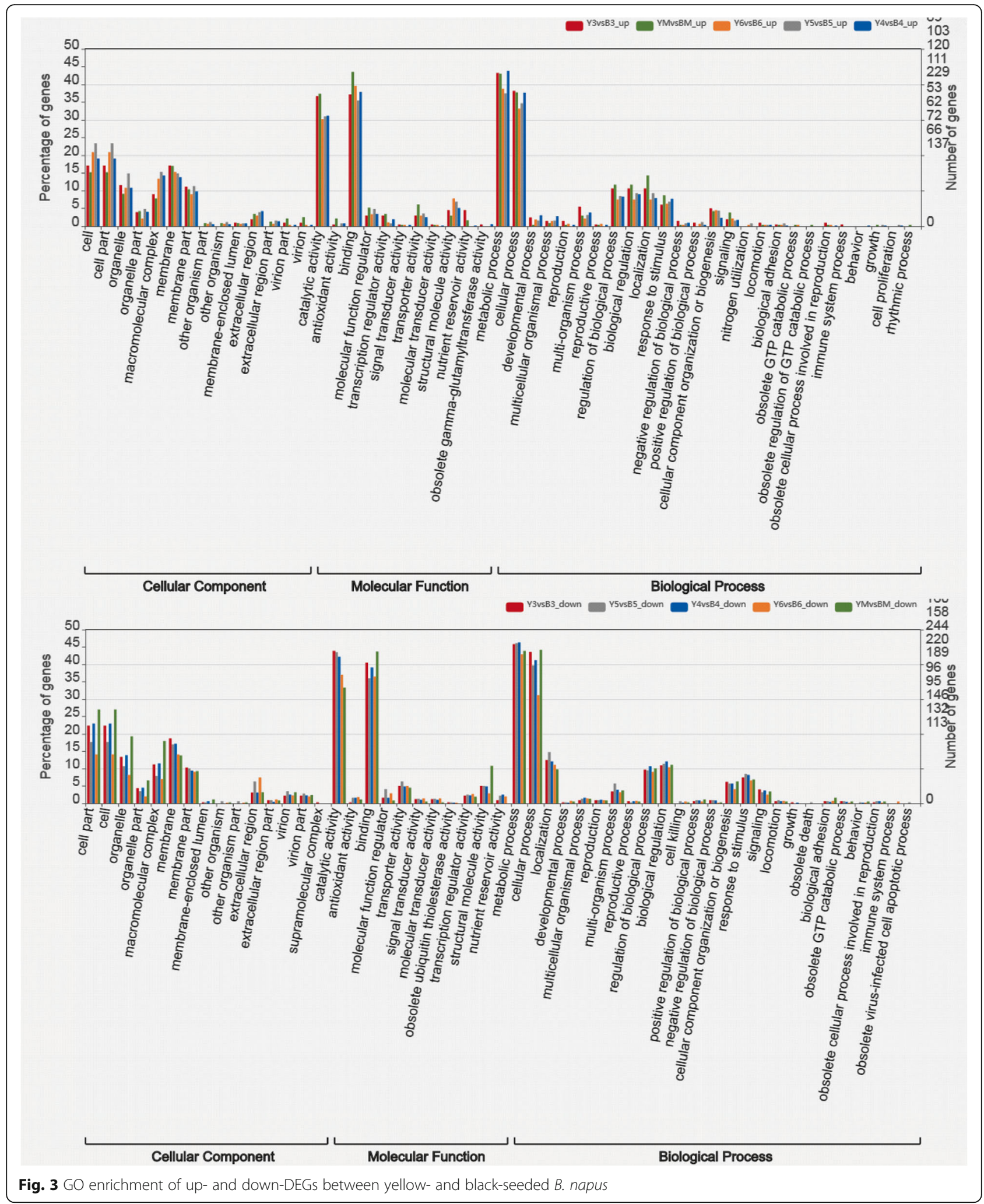

downregulated genes (1435 out of 1945 with GO annotations) between yellow and black rapeseed, 425, 39, 211, 89, and 65 DEGs were assigned to biosynthetic process (GO:
0009058), pigment metabolic process (GO: 0042440), oxidation-reduction process (GO: 0055114), cellular response to stimulus (GO: 0051716), and signal transduction 
(GO: 0007165), respectively. In contrast, 181 upregulated genes between yellow and black seed were assigned to biosynthetic process (GO: 0009058), 95 up-DEGs related to oxidation-reduction process (GO: 0055114), and 181 up-DEGs related to biosynthetic process (GO: 0009058).

KEGG pathway analysis showed that flavonoid biosynthesis was most significantly enriched with downregulated DEGs in comparisons of Y3 vs B3, Y4 vs B4, Y5 vs $\mathrm{B} 5$, and $\mathrm{Y} 6$ vs B6. Phenylpropanoid biosynthesis was also enriched with downregulated genes between the same stages of the two rapeseed lines, except for the mature stage. Phenylalanine metabolism, flavone and flavonol biosynthesis was enriched with downregulated DEGs in comparisons of $\mathrm{Y} 3$ vs B3 and Y4 vs B4 (Additional file 7: Figure S2). In contrast, the upregulated DEGs between yellow and black rapeseed were most significantly enriched in photosynthesis, followed by carbon metabolism, FA biosynthesis and metabolism (Additional file 8: Figure S3, Additional file 9: Table S6). The pathway enrichment analysis helps to determine the functions of DEGs and complemented secondary metabolisms that were not specified by GO terms. Using the $\log _{2}$ (Fold change) values between yellow and black rapeseed ( $\mathrm{Y} 3$ vs. B3, Y4 vs. B4, Y5 vs. B5, Y6 vs. B6, and YM vs. BM), we assigned all the DEGs to all the metabolism pathways using the Arabidopsis TAIR9 version as mapping reference. We also found many DEGs were enriched in metabolism (flavonoids, terpenes, phenylpropanoids and phenolics) and lipids (Additional file 10: Figure S4).

\section{DEGs associated with flavonoid and lignin biosynthesis}

As mentioned above, yellow seed color is associated with flavonoid and lignin biosynthesis. We found 77 DEGs related to phenylpropanoid and flavonoid biosynthesis and the phenylalanine metabolic pathway. As shown in Fig. 4, genes encoding 4-coumarate CoA ligase (4CL), cinnamate 4-hydroxylase $(\mathrm{C} 4 \mathrm{H})$, and phenylalanine ammonia-lyase (PAL) were downregulated in yellow rapeseed compared with black seed, except for a homolog of PAL (BnaC05g42780D). These genes play important roles in lignin biosynthesis, and the expression changes should be related to the reduced lignin in yellow seed. Genes involved in the flavonoid pathway were also downregulated in yellow seed compared to black seed, including $B A N$, CHI/TT5, DFR, F3H, FLS, LDOX, PAP, CHS/TT4, TT5, bHLH/TT8, WD40, and MYB, which participate in the biosynthesis of chalcones, flavanones, flavonols, anthocyanins, and PAs. However, we found a MATE (BnaC01g40630D) and UGT84A1 (BnaA01g18540D) were upregulated at the early developmental stages of yellow seed compared with black seed. Comparing with other two down-regulated MATEs in yellow-seeded B. napus, BnaA01g24940D (a homologous of AT3G03620) and BnaA07g18120D (a homologous of AtTT12), we found
BnaC01g40630D with lower expression throughout rapeseed development than that of BnaA01g24940D and BnaA07g18120D. Expressional differences of TCPs and CYPs were also identified, which might be related to flavonoid biosynthesis, since TCP could interact with R2R3-MYB [41], and CYPs (e.g., CYP71/75/93/81) have been reported to function in the biosynthesis of anthocyanin pigments and condensed tannins [42]. In addition, we compared the expression pattern of all the genes involved in secondary metabolism and found DEGs encoding peroxidase superfamily protein (BnaA02g02010D, BnaC06g32660D, BnaA02g14050D, and BnaC06g21080D), glycosyl hydrolase family protein (BnaUnng02770D), beta glucosidase 19/29/ 25 (BnaA06g09700D, BnaA05g04040D, BnaC05g11190D, BnaC01g43700D, and BnaC01g40610D), thioglucoside glucohydrolase 1 (BnaCnng53320D) were also assigned to phenylpropanoid biosynthesis and the phenylalanine metabolic pathway (Additional file 11: Figure S5). Generally, most of the genes involved in secondary metabolism were downregulated in yellow seed compared with black seed.

\section{DEGs associated with fatty acid biosynthesis and metabolism}

We found that genes involved in FA biosynthesis, metabolism, and degradation were also changed (Fig. 5). For instance, five up-DEGs in yellow seed were involved in FA biosynthesis, encoding an $\mathrm{NAD}(\mathrm{P})$-binding Rossmann-fold superfamily protein (BnaC01g40290D), a thioesterase superfamily protein (BnaA03g02830D), acetyl Coenzyme A carboxylase carboxyltransferase (BnaA03g17570D), stearoyl-acyl-carrier-protein desaturase (BnaA03g28050D), and chloroplastic acetylcoenzyme A carboxylase 1 (BnaC02g06560D). However, another homolog of the thioesterase superfamily protein (BnaC03g04180D) was downregulated in yellow seed compared with black seed. Five up-DEGs (BnaA03g02830D, BnaA03g17570D, BnaA03g28050D, BnaC01g40290D, and $\mathrm{BnaC02g06560D)}$ and six down-DEGs (BnaA02g30470D, BnaA05g30630D, BnaA08g28600D, BnaC02g38800D, Bna C03g04180D, and BnaC03g04350D) were involved in FA metabolism. BnaC03g04350D was also related to FA elongation. In addition, four down-DEGs (BnaC06g21150D, BnaA02g30470D, BnaA05g30630D, and BnaC02g38800D) were related to FA degradation. These DEGs might be related to the different oil content between yellow- and black-seeded B. napus.

\section{Real-time qPCR validates gene expression profiles}

To verify the sequencing results, DEGs involved in flavonoid and lignin biosynthesis, FA biosynthesis and metabolism were randomly selected for $\mathrm{qPCR}$ detection, including BnaC01g40290D encoding NAD(P)-binding Rossmann-fold superfamily protein/short-chain dehydrogenase/reductase (SDR), BnaA03g17570D encoding acetyl CoA carboxylase carboxyltransferase alpha subunit (CAC3), BnaC03g04350D 


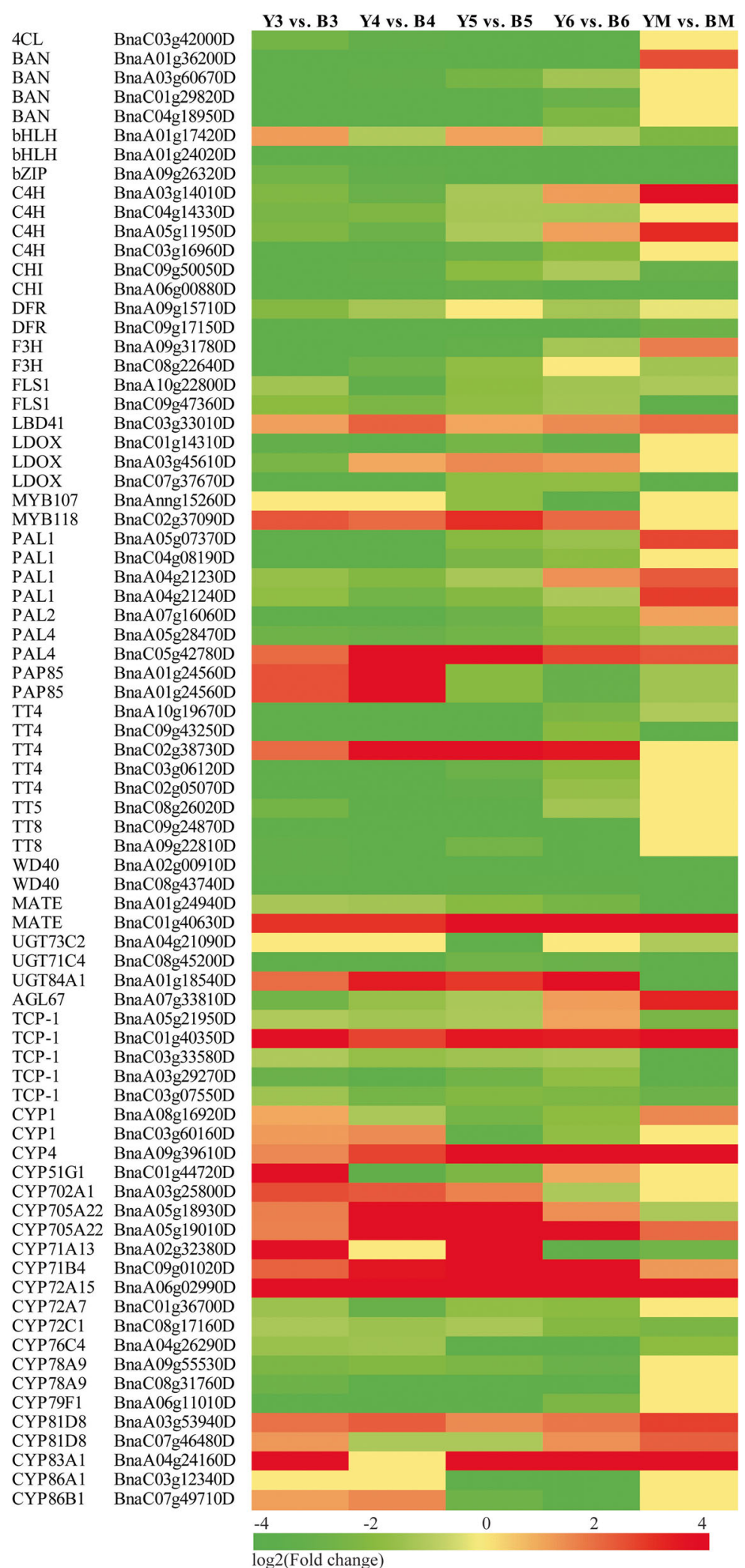

Fig. 4 Heatmap of DEGs related to flavonoid and lignin biosynthesis 


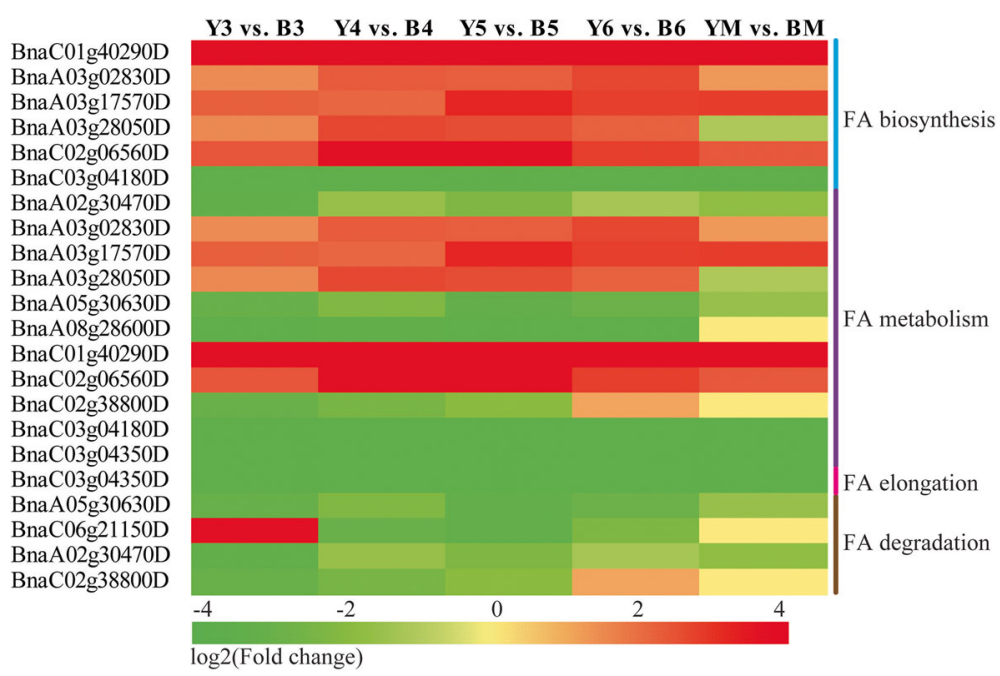

Fig. 5 Heatmap of DEGs related to fatty acid biosynthesis and metabolism

encoding 3-hydroxyacyl-CoA dehydratase PASTICCINO 2 (PAS2), 4CL (BnaC03g42000D), C4H (BnaC04g14330D, BnaA05g11950D), LBD41 (BnaC03g33010D), PAL (BnaC04g08190D), BAN (BnaA01g36200D), CHI (BnaC09g50050D), LDOX (BnaC01g14310D) and CHS (BnaA10g19670D). The results showed that the qPCR data of the DEGs were in accordance with the sequencing results throughout seed development of yellow- and black-seeded $B$. napus (Fig. 6).

\section{Discussion}

Due to the important economic value of rapeseed worldwide, research on improving seed quality has been carried out over the past half century, including reducing the erucic acid and glucosinolate contents and increasing the oleic acid content $[43,44]$. However, breeding of rapeseed has been greatly hindered by the narrow genetic background either due to the short history or the artificial selection [31]. Previously, Wang et al. created yellow-seeded $B$. napus from the backcrossing progenies of $B$. napus-S. alba hybrids, including $S$. alba specific fragments in the 38 chromosomes [8, 34, 45]. RNA-Seq is a reliable method in analyzing the gene expressional level on the whole transcriptome level, and it also helps to reveal the expressional differences among different samples, which could somehow explain the different characteristics among samples. In the past decade, RNA-Seq has been broadly applied in expressional comparison among plant samples, and in finding alternative splicing, novel transcripts, SNP and InDels [46-48]. To identify the gene expression differences related to yellow seed character and accompanying quality variations, we carried out comparative transcriptome analysis between different developmental stages of yellow- and black-seeded B. napus. As reported by Qu et al. (2013), accumulation of polymeric phenolic compounds were similar in yellow and black rapeseeds, but the flavonoids were more accumulated in black seed since $3 \mathrm{WAF}$ [49]. Thus, we collected the seeds at $3 \mathrm{WAF}, 4 \mathrm{WAF}, 5 \mathrm{WAF}$, $6 \mathrm{WAF}$ and mature stage for RNA-Seq analysis. We found a total of 39,632 SNPs in ten libraries, including 1142 SNPs common in five developmental stages of black seed and 1543 SNPs common in five developmental stages of yellow seed, indicating that genomic differences exist between yellow- and black-seeded B. napus. We found a total of 15,251 DEGs among all the libraries, including 80 upregulated and 151 downregulated genes identified throughout yellow and black seed development, which might be related to seed development and FA accumulation (Additional file 2: Table S2). Regarding the DEGs between yellow and black rapeseed, we found that most were annotated to biosynthetic, pigment metabolic, and oxidation-reduction processes, such as flavonoid and phenylpropanoid biosynthesis, phenylalanine metabolism, flavone and flavonol biosynthesis, and FA biosynthesis and metabolism (Additional file 8: Figure S3, Additional file 9: Table S6). This agreed with Hong et al. who found that the downregulated DEGs in yellow seed coats were enriched in phenylpropanoid and flavonoid biosynthesis using yellow- and brown-seeded near-isogenic lines (NILs) of rapeseed as research materials [50]. Similar expression changes in TT genes have been reported in B. juncea seed coat [51].

Flavonoid biosynthesis has been considered to be the main pathway related to plant pigmentation, and TT genes related to it have been clarified in A. thaliana. Recently, homologs of TTS in Brassica have been comprehensively identified by $\mathrm{Qu}$ et al. [3], as well as the expression patterns in different yellow- and black-seeded $B$. napus inbred lines. In the present study, we compared 


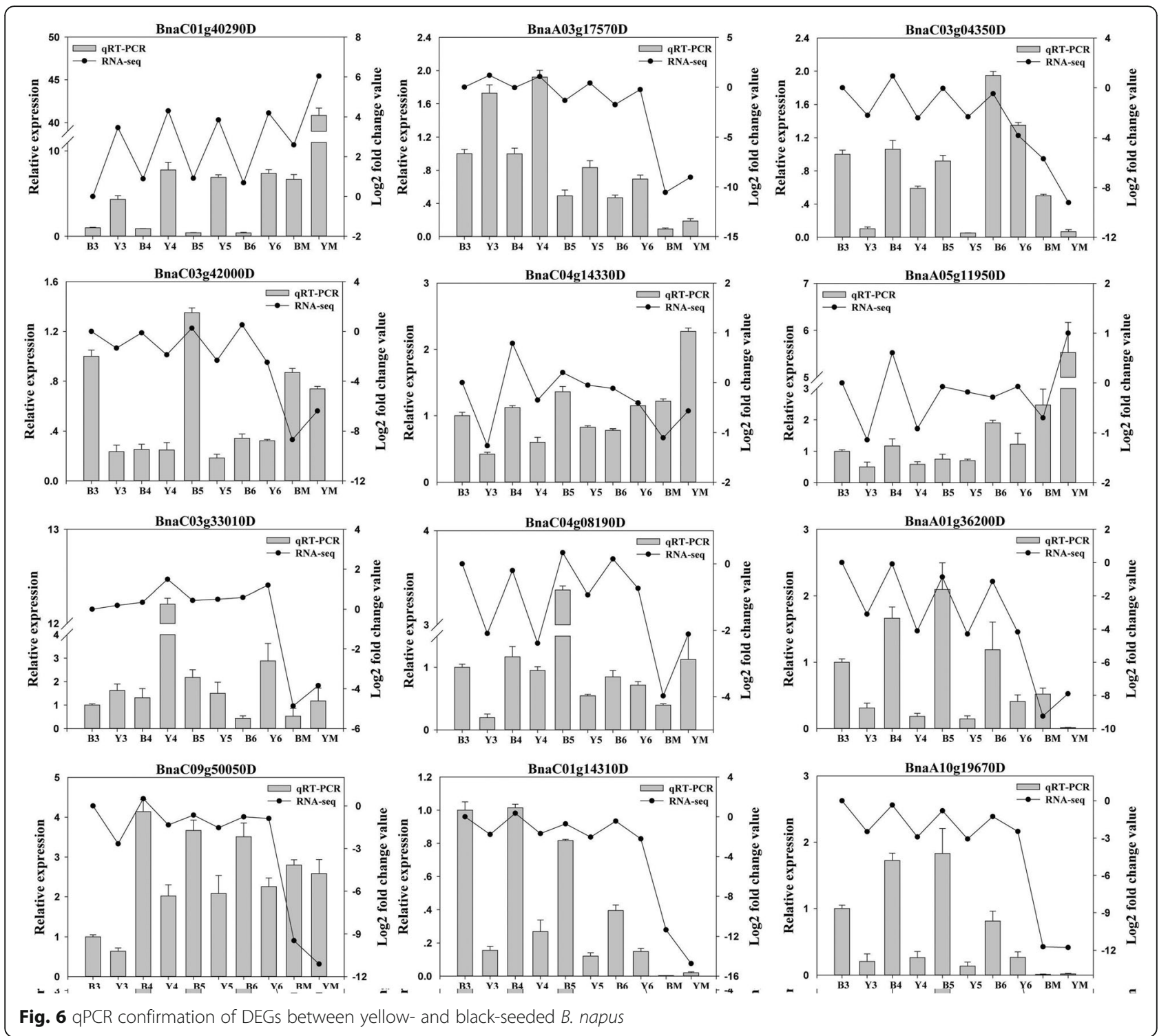

the expression pattern of genes related to flavonoid biosynthesis in developing seeds of B. napus cv. 'Yangyou 6' and yellow-seeded $B$. napus selected from somatic hybrids of B. napus-S. alba (Fig. 4). Four homologs of BnBAN (BnaA01g36200D, BnaA03g60670D, BnaC01g29820D and BnaC04g18950D) were downregulated at two to four consecutive developmental stages of yellow rapeseed compared with black rapeseed. Two homologs of BnDFR (BnaA09g15710D and BnaC09g17150D) were also expressed at lower levels in yellow seed, which encodes an enzyme catalyzing dihydroflavanones to leucoanthocyanidins that was then converted to anthocyanidin [52]. BnLDOX (BnaC01g14310D, BnaA03g45610D and BnaC07g37670D) was downregulated in yellow seed, which encodes leucoanthocyanidin reductase and catalyzes the formation of anthocyanidins. Genes encoding chalcone synthase (BnaA10g19670D, BnaC09g43250D,
BnaC03g06120D, BnaC02g05070D and BnaC08g26020D) were also downregulated throughout yellow seed development, which catalyzes the first step of flavonoid biosynthesis. BnTT5 (BnaC08g26020D) was also downregulated in yellow seed compared with black seed at specific stages, encoding chalcone isomerase that is redundant and responsive to lights in Brassicaceae [53]. BnTT8 (BnaC09g24870D and BnaA09g22810D) was also downregulated in yellow seed compared with black seed at specific stages, encoding the transcription factor bHLH as an important regulator throughout flavonoid biosynthesis. Another two bHLHs (BnaA01g17420D and BnaA01g24020D) were also downregulated, which have been proven to have functions in regulating jasmonate responses [54] and glucose homeostasis, and may affect ABA or salinity response in Arabidopsis [55]. In the present study, 21 CYPs were differentially expressed between yellow and black rapeseeds, although 
they were not shown to have functions in the secondary metabolism of B. napus. Previously, CYP71/75/93/81 have been reported to have functions in the biosynthesis of anthocyanin pigments and condensed tannins [42]. Lam et al. found that CYP75B4 and CYP93G1 promote tricin accumulation in Arabidopsis and O. sativa, which functions in generating 3 '-hydroxylated flavonoids and flavone aglycones $[56,57]$. Recently, Lei et al. found that a group of CYPs, including members of CYP71/72/77/78/81/85/86/ 90/93, were differentially expressed in Dendrobium catenatum from different locations and with different outlooks, which might be related to flavonoid biosynthesis [58]. CYP86 has been reported to be involved in suberin monomer biosynthesis in Arabidopsis [59]. Expressional differences of five TCPS were also identified, which might be related to flavonoid biosynthesis since TCP3 could interact with R2R3-MYB [41]. In addition, TCP1 directly activates DWARF4 and promotes brassinosteroid biosynthesis [60].

Asides from flavonoids, lignin has been revealed to have a positive correlation with seed coat color [26]. We found that $4 C L, C 4 H$ and $P A L$ were downregulated in yellow rapeseed compared with black seed, which play important roles in the less lignin in yellow seed (Fig. 4). PAL is involved in the first step of the phenylalanine metabolic pathway by catalyzing phenylalanine into cinnamic acid. Cinnamic acid was then transformed into coumaric acid by $\mathrm{C} 4 \mathrm{H}$. $4 \mathrm{CL}$ plays an important role in several steps of lignin biosynthesis and participates in the formation of coumaroyl-CoA, caffeoyl-CoA and feruloyl-CoA. Previously, Jiang et al. (2013) and Wang et al. (2018) reported that sinapic acid was more accumulated in yellow seed than black seed $[39,61]$. Down-regulation of 4CL in yellow rapeseed indicated that caffeic acid and ferulic acid might be less transformed into caffeoyl-CoA and feruloyl-CoA. Thus, sinapis acid as another downstream chemical of caffeic acid and ferulic acid was more accumulated (Fig. 7). All these compounds are necessary intermediates of lignin biosynthesis [5]. Hong et al. found that the expression of genes involved in lignin biosynthesis were slightly but not markedly changed in yellow-seeded NILs [50]. As to the other DEGs related to secondary metabolism, we found genes encoding S-adenosylmethionine synthetase 2 (BnaA09g00390D), peroxidase (BnaA02g14050D, BnaC06g32660D, BnaC06g21080D), branched-chain amino acid transaminase 1 (BnaA01 g36200D), methionine synthase 2 (BnaC03g33530D), aspartate aminotransferase 5 (BnaC01g06460D), phosphoglycerate kinase (BnaA07g34980D, BnaC02g46710D), lactate/malate dehydrogenase (BnaA09g16400D), glucose-1-phosphate adenylyltransferase family protein (BnaC04g33380D), beta glucosidase 19/28 (BnaA05g04030D, BnaC01g43700D, BnaC05g11190D, BnaA06g09700D), and pyruvate kinase (BnaA03g36910D) were downregulated in yellow rapeseed (Additional file 11: Figure S5). Peroxidase is one of three major enzymes involved in flavonoid oxidation through the associated reduction of hydrogen peroxide in the peroxidative cycle [62]. Hong et al. assigned peroxidase superfamily protein (e.g., peroxidase 4/7/25/52/53) to phenylpropanoid and flavonoid pathways, which were differentially expressed between yellow and black rapeseed NILs [50]. The DEGs related to flavonoid and lignin biosynthesis confirmed that they contribute to the different testa color.

Since the oil content of yellow seed was higher than that of black seed, we found 12 DEGs related to FA biosynthesis, metabolism, elongation and degradation

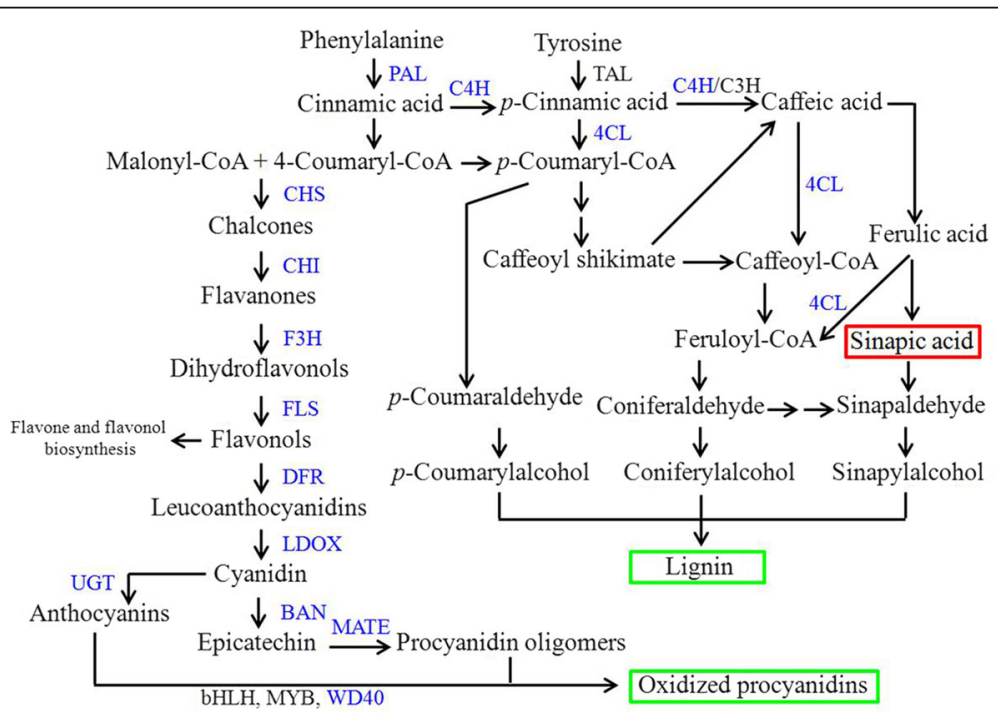

Fig. 7 Proposed model for DEGs related to yellow seed quality. Blue font, downregulated genes in yellow seed compared with black seed. Red frame, increased chemical in yellow seed. Green frame, decreased chemical in yellow seed 
(Fig. 5). In addition, we found that BnaA09g51510D (encoding pyruvate dehydrogenase E1 alpha) was upregulated throughout yellow seed development compared with black seed (Additional file 11: Figure S5). Its homo$\log$ in A. thaliana is important for seed oil biosynthesis. Plastidic pyruvate kinase (PKP) provides most of the pyruvate for plastidic FA synthesis, and the mutation of PKP severely impairs seed storage lipid synthesis [63]. BnaA03g17570D (acetyl Coenzyme A carboxylase carboxyltransferase alpha subunit) was upregulated in yellow seed. Its homolog in A. thaliana has been confirmed to be a subunit of heteromeric acetyl-CoA carboxylase (ACCase), which catalyzes the carboxyltransferase (CT) reaction. ACCase is responsible for the first step of FA synthesis [64]. Additionally, homolog of UDP-glucose pyrophosphorylase (UGP) (BnaA05g32480D), a sucrose-regulated protein, was upregulated in yellow seed, which is required for fumonisin B1-induced cell death [65]. A homolog of beta glucosidase 25 (BnaC01g40610D) was also upregulated in yellow seed, which is involved in the carbohydrate metabolic process [66]. Upregulation of cytochrome B5 reductase 1 (CB5R1) (BnaC02g02110D) in yellow seed might also be related to the FA variation, since CB5R is a microsomal membrane-bound protein that functions as part of the microsomal electron transfer system in FA desaturation [67]. CB5R can interact with ankyrin repeat-containing protein 2A (AKR2A), which interacts with ascorbate peroxidase 3 (APX3). APX3 can target peroxisomes [68]. This might be related to the above-mentioned expressional changes in genes encoding peroxidases. The expression changes of these genes may be helpful in explaining the FA variation in yellow seed and its adaptability to environments after flavonoid reduction.

\section{Conclusions}

In the present study, whole transcriptome gene expression was analyzed in developing seeds of yellow-seeded $B$. napus derived from hybrids of B. napus-S. alba, and black-seeded $B$. napus. We identified the DEGs with seed development, which might be related to the development and biosynthetic process. In addition, DEGs related to the quality difference between yellow and black rapeseed have been identified, which mainly participate in flavonoid biosynthesis, phenylpropanoid biosynthesis, phenylalanine metabolism, flavone and flavonol biosynthesis, fatty acid biosynthesis and metabolism. These down-regulated genes are helpful to explain the less pigmentation (e.g. CHS, CHI, F3H, FLS, DFR, LDOC, BAN) and lignin (PAL, C4H and $4 C L$ ), and higher oil content in yellow rapeseed compared with black seed (Fig. 7). Future functional analysis of these genes would contribute to the molecular dissection of yellow seed character in B. napus.

\section{Methods}

\section{Plant material}

Yellow-seeded B. napus (line W82) was preserved in our lab and was selected from back-crossing progenies of somatic hybrids of $B$. napus-S. alba. The black-seeded rapeseed (B. napus cv. 'Yangyou 6') was obtained from the Jiangsu Lixiahe Region Agricultural Research Institute, China [45]. Both rapeseed lines were grown in the experimental field of Yangzhou University, Yangzhou, China. In addition to the visible difference in seed coat color, the flavonoid content in yellow seed was lower than that in black seed, and the seed FA composition and content were different between yellow- and black-seeded B. napus. The oil content of W82 was 6\% higher than that of Yangyou 6. Higher protein and sucrose contents, less dietary fiber and crude fiber, and fewer glucosinolates accumulated in the seed meal of yellow rapeseed compared with black rapeseed (Additional file 12: Table S7) [8, 39, 61, 69]. The developing seeds at $3 \mathrm{WAF}, 4 \mathrm{WAF}, 5 \mathrm{WAF}, 6 \mathrm{WAF}$, and mature seeds were collected from three pods each of ten individual plants for comparative analysis. During the seed development, differentially accumulated pigments were visual since 5 WAF of yellow and black rapeseeds. Proanthocyanidins (PAs) were less accumulated throughout yellow seed development than black seed $[39,70]$.

RNA extraction, library construction, and RNA sequencing For each developmental stage of each rapeseed line, five RNA samples were separately isolated from polled seeds of ten plants using Trizol Reagent Solution (Invitrogen, USA). Each library was pooled by mixing equal quantities of five RNA samples. The RNA quality was validated using agarose gel electrophoresis, Nanodrop, Qubit, and Agilent 2100 to confirm the purity, concentration, and integrity, respectively. mRNA was purified using beads with Oligo (dT), and cDNA was then synthesized with random hexamers after fragmentation of mRNA. After purification of cDNA with AMPure XP beads, unique adaptors and indexes were ligated. Certain fragments were selected with beads and enriched using PCR amplification. Finally, ten cDNA libraries for five developmental stages (3 WAF, 4 WAF, 5 WAF, 6 WAF and mature seeds) of yellow- and black-seeded $B$. napus were normalized based on a Qubit assessment, and the insert size was validated by Agilent 2100 . Then, the polled libraries were sequenced by the Illumina HiSeq ${ }^{\mathrm{Tm}}$ 2000 platform.

\section{Bioinformatics analysis}

After removing adaptor sequences and low-quality sequences, clean reads were mapped to the $B$. napus $\mathrm{cv}$. Darmor-bzh genome (version 5) using TopHat2 [71, 72]. 
Based on the predicted gene models of Cufflinks, classification and statistics of alternative splicing were carried out using AS profile [73, 74]. Novel transcripts were predicted using Cufflinks to assemble the mapped reads on the genome and compare it with the known gene models [75]. The abundance of reads mapped to reference was estimated and normalized using Reads Per Kilo bases per Million reads (RPKM), and all the transcripts with RPKM value $>1$ were used for further analysis. Differential gene expression analysis was performed using DESeq $[76,77]$, and DEGs between libraries were screened with a threshold of $\mid$ (FoldChange) $\mid>1$ and $q$ value $<0.005$. All the DEGs among different libraries were clustered base on the $\log _{10}(\mathrm{RPKM}+1)$ and $\log _{2}$ (Fold Change) value. Gene ontology (GO) enrichment of the DEGs was performed using GOseq with a corrected $p$-value $<0.05$ [78], and KEGG pathway enrichment of DEGs was performed using KOBAS 2.0 with a corrected $p$-value $<0.05$ [79]. An overview of pathways related to these DEGs was predicted by MapMan (version 3.6.0) analysis [80].

\section{CDNA synthesis and qRT-PCR validation}

Subsamples of RNA-Seq were reverse transcribed into cDNA for real-time qPCR validation using the Revert Aid First Strand cDNA Synthesis Kit and SYBR Green Real-Time PCR Master Mixes (Thermo, USA). qRT-PCR was performed on a fluorescence quantitative system Mx3005P (Agilent, USA). Genes, primers and size of the amplicon are listed in Additional file 13: Table S8. B. napus $\beta$-actin (NCBI AF111812) was used as an endogenous control to generate the $\Delta \mathrm{Ct}$ for three technological replicates.

\section{Additional files}

Additional file 1: Table S1. Summary of read mapping in RNA-Seq analysis of yellow and black rapeseeds. B3, B4, B5, B6 and BM indicate $3 \sim 6$ WAF and mature black seed. Y3, Y4, Y5, Y6 and YM indicate $3 \sim 6$ WAF and mature yellow seed. (XLSX $34577 \mathrm{~kb}$ )

Additional file 2: Table S2. DEGs identified with black or yellow seed development and between same developmental stages of two rapeseeds. (XLSX $11030 \mathrm{~kb}$ )

Additional file 3: Table S3. DEGs related to seed development. (XLSX $94 \mathrm{~kb})$

Additional file 4: Table S4. Up- and downregulated genes among all the developmental stages of yellow- and black-seeded B. napus. (XLSX $25 \mathrm{~kb}$ )

Additional file 5 Figure S1. The SOM clusters of DEGs from five developmental stages of two rapeseed lines. (XLSX $173 \mathrm{~kb}$ )

Additional file 6: Table S5. All the up- and down-DEGs with GO annotation. (XLSX $63 \mathrm{~kb}$ )

Additional file 7: Figure S2. KEGG enrichment of DEGs ( $Y 3$ vs. B3) in flavonoid and phenylpropanoid biosynthesis. (XLSX $24 \mathrm{~kb}$ )

Additional file 8: Figure S3. KEGG enrichment of up- and down-DEGs between yellow- and black-seeded B. napus. (XLS 31 kb)
Additional file 9: Table S6. KEGG pathways of DEGs between yellowand black-seeded B. napus. (PDF 519 kb)

Additional file 10: Figure S4. Overview of pathways related to the DEGs between yellow- and black-seeded B. napus. (PDF 981 kb)

Additional file 11: Figur S5. Heatmap of DEGs involved in secondary metabolism. (JPG $6164 \mathrm{~kb}$ )

Additional file 12: Table S7. Quality differences between yellow- and black-seeded B. napus. (JPG 1896 kb)

Additional file 13: Table S8. Primers for $\mathrm{QPCR}$ validation of DEGs (JPG $3234 \mathrm{~kb})$

\section{Abbreviations}

4CL: 4-coumarate CoA ligase; ACCase: Acetyl-CoA carboxylase; AKR2A: Ankyrin repeat-containing protein 2A; APX3: Ascorbate peroxidase; C4H: Cinnamate 4-hydroxylase; CB5R1: Cytochrome B5 reductase 1; CT: Carboxyltransferase; CYP: Cytochrome P450; DEG: Differentially expressed gene; EBG: Early biosynthesis gene; FA: Fatty acid; GO: Gene ontology; GWAS: Genome wide association study; KEGG: Kyoto encyclopedia of genes and genomes; LBG: Late biosynthesis gene; LEA: Late embryogenesis abundant hydroxyproline; MBW: MYB-bHLH-WD40; NIL: Near-isogenic line; PA: Proanthocyanin; PAL: Phenylalanine ammonia-lyase; PKP: Pyruvate kinase; qRT-PCR: Quantitative real-time polymerase chain reaction; RPKM: Reads per kilo bases per million reads; SNP: Single nucleotide polymorphism; TT: Transparent testa; UGP: UDP-Glucose pyrophosphorylase; WAF: Weeks after flowering

\section{Acknowledgements \\ Not applicable.}

\section{Funding}

This study was supported by the National Key Basic Research Program of China (2015CB150201), the National Natural Science Foundations (31330057, $31771825,31401414)$, the National Key Research and Development Program of China (2016YFD0101000, 2016YFD0102000), the Natural Science Foundation of Jiangsu Province (BK20180101, BK20140478), China Postdoctoral Science Foundation (2015 T80591, 2014 M561719), Jiangsu Postdoctoral Science Foundation (1401078B), the Postgraduate Research \& Practice Innovation Program of Jiangsu Province (XKYCX17 066), the Priority Academic Program Development of Jiangsu Higher Education Institutions, and Yangzhou University for Excellent Talent Support Program. The founders did not play any roles in the design, analysis, interpretation of this study or relevant data.

Availability of data and materials

All the data pertaining to the present study have been included in the tables and figures of the manuscript, and the authors are pleased to share all the data and plant materials upon reasonable request.

\section{Authors' contributions}

JJ performed the experiments, analyzed the RNA-seq data, and drafted the manuscript. SZ, YY, YW and LZ sampled the materials and performed QRTPCR analysis. JB and YPW revised the manuscript. All the authors approved the final manuscript.

\section{Ethics approval and consent to participate}

This study has not directly involved humans or animals. The yellow-seeded $B$ napus, which originated from somatic hybridization between $B$. napus and $S$. alba, was created and preserved by our group. The black-seeded B. napus was obtained from the Jiangsu Lixiahe Region Agricultural Research Institute, China. No specific permission was required for use of these materials for experimental purposes. The seedlings were grown in the experimental field of Yangzhou University, Yangzhou, China as per standard practices, and samples were harvested at the required time. We comply with the Convention on the Trade in Endangered Species of Wild Fauna and Flora.

Consent for publication

Not applicable.

Competing interests

The authors declare that they have no competing interests. 


\section{Publisher's Note}

Springer Nature remains neutral with regard to jurisdictional claims in published maps and institutional affiliations.

\section{Author details \\ ${ }^{1}$ Jiangsu Provincial Key Laboratory of Crop Genetics and Physiology, Yangzhou University, Yangzhou 225009, China. ${ }^{2}$ School of Biological Sciences, University of Western Australia, Perth, WA, Australia.}

\section{Received: 1 November 2018 Accepted: 7 May 2019} Published online: 16 May 2019

\section{References}

1. Wittkop B, Snowdon RJ, Friedt W. New NIRS calibrations for fiber fractions reveal broad genetic variation in Brassica napus seed quality. J Agric Food Chem. 2012;60(9):2248-56.

2. Abbadi A, Leckband G. Rapeseed breeding for oil content, quality, and sustainability. Eur J Lipid Sci Technol. 2011;113(10):1198-206.

3. Qu C, Zhao H, Fu F, Wang Z, Zhang K, Zhou Y, Wang X, Wang R, Xu X, Tang $Z$, et al. Genome-wide survey of flavonoid biosynthesis genes and gene expression analysis between black- and yellow-seeded Brassica napus. Front Plant Sci. 2016;7:1755.

4. Lepiniec L, Debeaujon I, Routaboul JM, Baudry A, Pourcel L, Nesi N, Caboche M. Genetics and biochemistry of seed flavonoids. Annu Rev Plant Biol. 2006:57:405-30.

5. Liu Q, Luo L, Zheng L. Lignins: biosynthesis and biological functions in plants. Int J Mol Sci. 2018;19(2):335-50.

6. Mittasch J, Bottcher C, Frolov A, Strack D, Milkowski C. Reprogramming the phenylpropanoid metabolism in seeds of oilseed rape by suppressing the orthologs of reduced epidermal fluorescence1. Plant Physiol. 2013;161(4): 1656-69.

7. Rahman $\mathrm{MH}$, Joersbo M, Poulsen MH. Production of yellow-seeded Brassica napus of double low quality. Plant Breed. 2001;120:473-8.

8. Li A, Wei C, Jiang J, Zhang Y, Snowdon RJ, Wang Y. Phenotypic variation in progenies from somatic hybrids between Brassica napus and Sinapis alba. Euphytica. 2009;170(3):289-96.

9. Appelhagen I, Thiedig K, Nordholt N, Schmidt N, Huep G, Sagasser M, Weisshaar B. Update on transparent testa mutants from Arabidopsis thaliana: characterisation of new alleles from an isogenic collection. Planta. 2014; 240(5):955-70.

10. Xu W, Grain D, Bobet S, Le Gourrierec J, Thevenin J, Kelemen Z, Lepiniec L, Dubos C. Complexity and robustness of the flavonoid transcriptional regulatory network revealed by comprehensive analyses of MYB-bHLH-WDR complexes and their targets in Arabidopsis seed. New Phytol. 2014;202(1): 132-44.

11. Appelhagen I, Jahns O, Bartelniewoehner L, Sagasser M, Weisshaar B, Stracke R. Leucoanthocyanidin Dioxygenase in Arabidopsis thaliana: characterization of mutant alleles and regulation by MYB-BHLH-TTG1 transcription factor complexes. Gene. 2011;484(1-2):61-8.

12. Xu BB, Li JN, Zhang XK, Wang R, Xie LL, Chai YR. Cloning and molecular characterization of a functional flavonoid 3'-hydroxylase gene from Brassica napus. J Plant Physiol. 2007;164(3):350-63.

13. Wei YL, Li JN, Lu J, Tang ZL, Pu DC, Chai YR. Molecular cloning of Brassica napus TRANSPARENT TESTA 2 gene family encoding potential MYB regulatory proteins of proanthocyanidin biosynthesis. Mol Biol Rep. 2007; 34(2):105-20.

14. Ni Y, Jiang H-L, Lei B, Li J-N, Chai Y-R. Molecular cloning, characterization and expression of two rapeseed (Brassica napus L.) cDNAs orthologous to Arabidopsis thaliana phenylalanine ammonialyase 1. Euphytica. 2007;159(1-2):1-16.

15. Chai YR, Lei B, Huang HL, Li JN, Yin JM, Tang ZL, Wang R, Chen L. TRANSPARENT TESTA 12 genes from Brassica napus and parental species: cloning, evolution, and differential involvement in yellow seed trait. Mol Gen Genomics. 2009;281(1):109-23.

16. Auger B, Baron C, Lucas MO, Vautrin S, Berges H, Chalhoub B, Fautrel A, Renard M, Nesi N. Brassica orthologs from BANYULS belong to a small multigene family, which is involved in procyanidin accumulation in the seed. Planta. 2009;230(6):1167-83.

17. Lu J, Li JN, Lei B, Wang SG, Chai YR. Molecular cloning and characterization of two Brassica napus TTG1 genes reveal genus-specific nucleotide preference, extreme protein-level conservation and fast divergence of organ-specificity. Genes Genomics. 2009;31:129-42.

18. Zhang K, Lu K, Qu C, Liang Y, Wang R, Chai Y, Li J. Gene silencing of BnTT10 family genes causes retarded pigmentation and lignin reduction in the seed coat of Brassica napus. PLoS One. 2013;8(4):e61247.

19. Lian J, Lu X, Yin N, Ma L, Lu J, Liu X, Li J, Lu J, Lei B, Wang R, et al. Silencing of BnTT1 family genes affects seed flavonoid biosynthesis and alters seed fatty acid composition in Brassica napus. Plant Sci. 2017;254:32-47.

20. Yu CY. Molecular mechanism of manipulating seed coat coloration in oilseed Brassica species. J Appl Genet. 2013;54(2):135-45.

21. Kebede B, Cheema K, Greenshields DL, Li C, Selvaraj G, Rahman H. Construction of genetic linkage map and mapping of QTL for seed color in Brassica rapa. Genome. 2012;55(12):813-23.

22. Rahman M, McVetty PB, Li G. Development of SRAP, SNP and multiplexed SCAR molecular markers for the major seed coat color gene in Brassica rapa L. Theor Appl Genet. 2007;115(8):1101-7.

23. Zhao H, Basu U, Kebede B, Qu C, Li J, Rahman H. Fine mapping of the major QTL for seed coat color in Brassica rapa var. yellow Sarson by use of NIL populations and transcriptome sequencing for identification of the candidate genes. PLoS One. 2019;14(2):e0209982.

24. Mahmood T, Rahman MH, Stringam GR, Raney JP, Good AG. Molecular markers for seed colour in Brassica juncea. Genome. 2005;48(4):755-60.

25. Mahmood T, Rahman MH, Stringam GR, Yeh F, Good AG. Identification of quantitative trait loci (QTL) for oil and protein contents and their relationships with other seed quality traits in Brassica juncea. Theor Appl Genet. 2006;113:1211-20.

26. Liu L, Stein A, Wittkop B, Sarvari P, Li J, Yan X, Dreyer F, Frauen M, Friedt W, Snowdon RJ. A knockout mutation in the lignin biosynthesis gene CCR 1 explains a major QTL for acid detergent lignin content in Brassica napus seeds. Theor Appl Genet. 2012;124(8):1573-86.

27. Stein A, Wittkop B, Liu L, Obermeier C, Friedt W, Snowdon RJ, Chevre AM. Dissection of a major QTL for seed colour and fibre content in Brassica napus reveals colocalization with candidate genes for phenylpropanoid biosynthesis and flavonoid deposition. Plant Breed. 2013:132(4):382-9.

28. Wang J, Xian X, Xu X, Qu C, Lu K, Li J, Liu L. Genome-wide association mapping of seed coat color in Brassica napus. J Agric Food Chem. 2017; 65(26):5229-37.

29. Jaakola L. New insights into the regulation of anthocyanin biosynthesis in fruits. Trends Plant Sci. 2013;18(9):477-83.

30. Chalhoub B, Denoeud F, Liu S, Parkin IA, Tang H, Wang X, Chiquet J, Belcram H, Tong C, Samans B, et al. Plant genetics. Early allopolyploid evolution in the post-Neolithic Brassica napus oilseed genome. Science. 2014:345(6199):950-3

31. Wei D, Cui Y, He Y, Xiong Q, Qian L, Tong C, Lu G, Ding Y, Li J, Jung C, et al. A genome-wide survey with different rapeseed ecotypes uncovers footprints of domestication and breeding. J Exp Bot. 2017;68(17):4791-801.

32. Rashid A, Rakov G, Downey RK. Development of yellow seeded Brassica napus through interspecific crosses. Plant Breed. 1994;112:127-34.

33. Wen J, Zhu L, Qi L, Ke H, Yi B, Shen J, Tu J, Ma C, Fu T. Characterization of interploid hybrids from crosses between Brassica juncea and B. oleracea and the production of yellow-seeded B. napus. Theor Appl Genet. 2012;125:19-32.

34. Wang YP, Zhao XX, Sonntag K, Wehling P, Snowdon RJ. Behaviour of Sinapis alba chromosomes in a Brassica napus background revealed by genomic insitu hybridization. Chromosom Res. 2005;13(8):819-26.

35. Jiang JJ, Zhao XX, Tian W, Li TB, Wang YP. Intertribal somatic hybrids between Brassica napus and Camelina sativa with high linolenic acid content. Plant Cell, Tissue and Organ Culture (PCTOC). 2009;99(1):91-5.

36. Warwick SI, Simard MJ, Legere A, Beckie HJ, Braun L, Zhu B, Mason P, Seguin-Swartz G, Stewart CN, Jr.: Hybridization between transgenic Brassica napus L. and its wild relatives: Brassica rapa L., Raphanus raphanistrum L., Sinapis arvensis L., and Erucastrum gallicum (Willd.) O.E. Schulz. Theor Appl Genet 2003, 107(3):528-539.

37. Zhang L, Jiang S, Xin R, Zhang F, Wang J, Guan R. Obtaining of yellowseeded rapeseed germplasm from intergeneric hybridization between Brassica napus and Descurainia sophia. Chinese Journal of Oil Crop Sciences. 2009;31:434-9.

38. Wang Y, Sonntag K, Rudloff E, Chen J. Intergeneric somatic hybridization between Brassica napus L. and Sinapis alba L. J Integr Plant Biol. 2005;47(1):8.

39. Jiang J, Shao Y, Li A, Lu C, Zhang Y, Wang Y. Phenolic composition analysis and gene expression in developing seeds of yellow- and black-seeded Brassica napus. J Integr Plant Biol. 2013;55(6):537-51. 
40. Jiang J, Shao Y, Li A, Zhang Y, Wei C, Wang Y. FT-IR and NMR study of seed coat dissected from different colored progenies of Brassica napus-Sinapis alba hybrids. J Sci Food Agric. 2013;93(8):1898-902.

41. Li S, Zachgo S. TCP3 interacts with R2R3-MYB proteins, promotes flavonoid biosynthesis and negatively regulates the auxin response in Arabidopsis thaliana. Plant J. 2013:76(6):901-13.

42. Ayabe S, Akashi T. Cytochrome P450s in flavonoid metabolism. Phytochem Rev. 2006;5(2-3):271-82

43. Wu G, Wu Y, Xiao L, Li X, Lu C. Zero erucic acid trait of rapeseed (Brassica napus L.) results from a deletion of four base pairs in the fatty acid elongase 1 gene. Theor Appl Genet. 2008;116(4):491-9.

44. Schierholt A, Becker HC, Ecke W. Mapping a high oleic acid mutation in winter oilseed rape (Brassica napus L). Theor Appl Genet. 2000;101(5-6):897-901.

45. Li A, Jiang J, Zhang Y, Snowdon RJ, Liang G, Wang Y. Molecular and cytological characterization of introgression lines in yellow seed derived from somatic hybrids between Brassica napus and Sinapis alba. Mol Breed. 2012:29:209-19.

46. Zhu J, Wang X, Xu Q, Zhao S, Tai Y, Wei C. Global dissection of alternative splicing uncovers transcriptional diversity in tissues and associates with the flavonoid pathway in tea plant (Camellia sinensis). BMC Plant Biol. 2018; 18(1):266.

47. Zhang G, Sun M, Wang J, Lei M, Li C, Zhao D, Huang J, Li W, Li S, Li J, et al. PacBio full-length cDNA sequencing integrated with RNA-seq reads drastically improves the discovery of splicing transcripts in rice. Plant J. 2019;97(2):296-305.

48. Thakur O, Randhawa GS. Identification and characterization of SSR, SNP and InDel molecular markers from RNA-Seq data of guar (Cyamopsis tetragonolobaL Taub) roots. BMC genomics. 2018;19(1):951.

49. Qu C, Fu F, Lu K, Zhang K, Wang R, Xu X, Wang M, Lu J, Wan H, Zhanglin T, et al. Differential accumulation of phenolic compounds and expression of related genes in black- and yellow-seeded Brassica napus. J Exp Bot. 2013; 64(10):2885-98.

50. Hong M, Hu K, Tian T, Li X, Chen L, Zhang Y, Yi B, Wen J, Ma C, Shen J, et al. Transcriptomic analysis of seed coats in yellow-seeded Brassica napus reveals novel genes that influence proanthocyanidin biosynthesis. Front Plant Sci. 2017:8:1674.

51. Liu X, Lu Y, Yuan Y, Liu S, Guan C, Chen S, Liu Z. De novo transcriptome of Brassica juncea seed coat and identification of genes for the biosynthesis of flavonoids. PLoS One. 2013;8(8):e71110.

52. Wang H, Fan W, Li H, Yang J, Huang J, Zhang P. Functional characterization of Dihydroflavonol-4-reductase in anthocyanin biosynthesis of purple sweet potato underlies the direct evidence of anthocyanins function against abiotic stresses. PLoS One. 2013;8(11):e78484.

53. Zhou B, Wang Y, Zhan Y, Li Y, Kawabata S. Chalcone synthase family genes have redundant roles in anthocyanin biosynthesis and in response to blue/ UV-A light in turnip (Brassica rapa; Brassicaceae). Am J Bot. 2013;100(12): 2458-67.

54. Sasaki-Sekimoto Y, Saito H, Masuda S, Shirasu K, Ohta H. Comprehensive analysis of protein interactions between JAZ proteins and bHLH transcription factors that negatively regulate jasmonate signaling. Plant Signal Behav. 2014;9(1):e27639.

55. Min JH, Ju HW, Yoon D, Lee KH, Lee S, Kim CS. Arabidopsis basic helix-loophelix 34 (bHLH34) is involved in glucose signaling through binding to a GAGA Cis-element. Front Plant Sci. 2017:8:2100.

56. Lam PY, Zhu FY, Chan WL, Liu H, Lo C. Cytochrome P450 $93 \mathrm{G} 1$ is a flavone synthase II that channels flavanones to the biosynthesis of tricin O-linked conjugates in rice. Plant Physiol. 2014;165(3):1315-27.

57. Lam PY, Liu H, Lo C: Completion of Tricin biosynthesis pathway in Rice: cytochrome P450 75B4 is a unique Chrysoeriol 5'-hydroxylase. Plant Physiol 2015, 168(4):1527-1536.

58. Lei Z, Zhou C, Ji X, Wei G, Huang Y, Yu W, Luo Y, Qiu Y. Transcriptome analysis reveals genes involved in flavonoid biosynthesis and accumulation in Dendrobium catenatum from different locations. Sci Rep. 2018;8(1):6373.

59. Ma B, Luo Y, Jia L, Qi X, Zeng Q, Xiang Z, He N. Genome-wide identification and expression analyses of cytochrome P450 genes in mulberry (Morus notabilis). J Integr Plant Biol. 2014;56(9):887-901.

60. Li S. Transcriptional control of flavonoid biosynthesis: fine-tuning of the MYB-bHLH-WD40 (MBW) complex. Plant Signal Behav. 2014;9(1):e27522.

61. Wang Y, Meng G, Chen S, Chen Y, Jiang J, Wang YP. Correlation analysis of phenolic contents and antioxidation in yellow- and black-seeded Brassica napus. Molecules. 2018;23:1815-29.
62. Pourcel L, Routaboul JM, Cheynier V, Lepiniec L, Debeaujon I. Flavonoid oxidation in plants: from biochemical properties to physiological functions. Trends Plant Sci. 2007;12(1):29-36.

63. Lonien J, Schwender J. Analysis of metabolic flux phenotypes for two Arabidopsis mutants with severe impairment in seed storage lipid synthesis. Plant Physiol. 2009;151(3):1617-34.

64. Keereetaweep J, Liu H, Zhai Z, Shanklin J. Biotin attachment domaincontaining proteins irreversibly inhibit acetyl CoA carboxylase. Plant Physiol. 2018;177(1):208-15.

65. Chivasa S, Tome DF, Slabas AR. UDP-glucose pyrophosphorylase is a novel plant cell death regulator. J Proteome Res. 2013;12(4):1743-53.

66. Xu Z, Escamilla-Trevino L, Zeng L, Lalgondar M, Bevan D, Winkel B, Mohamed A, Cheng CL, Shih MC, Poulton J, et al. Functional genomic analysis of Arabidopsis thaliana glycoside hydrolase family 1. Plant Mol Biol. 2004:55(3):343-67.

67. Fukuchi-Mizutani M, Mizutani M, Tanaka Y, Kusumi T, Ohta D. Microsomal electron transfer in higher plants: cloning and heterologous expression of $\mathrm{NADH}$-cytochrome b5 reductase from Arabidopsis. Plant Physiol. 1999;119(1): 353-62.

68. Shen G, Kuppu S, Venkataramani S, Wang J, Yan J, Qiu X, Zhang H. ANKYRIN REPEAT-CONTAINING PROTEIN 2A is an essential molecular chaperone for peroxisomal membrane-bound ASCORBATE PEROXIDASE3 in Arabidopsis. Plant Cell. 2010;22(3):811-31.

69. Jiang J, Wang $Y$, Xie T, Rong H, Li A, Fang Y, Wang Y. Metabolic characteristics in meal of black rapeseed and yellow-seeded progeny of Brassica napus-Sinapis alba hybrids. Molecules. 2015:20(12):21204-13.

70. Wang Y, Rong H, Xie T, Jiang J, Wu J, Wang Y. Comparison of DNA methylation in the developing seeds of yellow- and black-seeded Brassica napus through MSAP analysis. Euphytica. 2016;209:157-69.

71. Kim D, Pertea G, Trapnell C, Pimentel H, Kelley R, Salzberg SL. TopHat2: accurate alignment of transcriptomes in the presence of insertions, deletions and gene fusions. Genome Biol. 2013;14(4):R36.

72. Jiang L, Schlesinger F, Davis CA, Zhang Y, Li R, Salit M, Gingeras TR, Oliver B. Synthetic spike-in standards for RNA-seq experiments. Genome Res. 2011; 21(9):1543-51.

73. Trapnell C, Pachter L, Salzberg SL. TopHat: discovering splice junctions with RNA-Seq. Bioinformatics. 2009:25(9):1105-11.

74. Ghosh S, Chan CK. Analysis of RNA-Seq data using TopHat and cufflinks. Methods Mol Biol. 2016;1374:339-61.

75. Trapnell C, Williams BA, Pertea G, Mortazavi A, Kwan G, van Baren MJ, Salzberg SL, Wold BJ, Pachter L. Transcript assembly and quantification by RNA-Seq reveals unannotated transcripts and isoform switching during cell differentiation. Nat Biotechnol. 2010;28(5):511-5.

76. Mortazavi A, Williams BA, McCue K, Schaeffer L, Wold B. Mapping and quantifying mammalian transcriptomes by RNA-Seq. Nat Methods. 2008;5(7): $621-8$.

77. Anders S, Huber W. Differential expression analysis for sequence count data. Genome Biol. 2010;11(10):R106.

78. Young MD, Wakefield MJ, Smyth GK, Oshlack A. Gene ontology analysis for RNA-seq: accounting for selection bias. Genome Biol. 2010;11(2):R14.

79. Mao XCT, Olyarchuk JG, Wei L. Automated genome annotation and pathway identification using the KEGG Orthology (KO) as a controlled vocabulary. Bioinformatics. 2005;21(19):3787-93.

80. Usadel B, Poree F, Nagel A, Lohse M, Czedik-Eysenberg A, Stitt M. A guide to using MapMan to visualize and compare Omics data in plants: a case study in the crop species, maize. Plant Cell Environ. 2009;32(9):1211-29. 